\title{
Simplified diagnostic procedure for obstructive sleep apnoea syndrome: lower subsequent compliance with CPAP
}

\author{
J. Krieger, E. Sforza, C. Petiau, T. Weiss
}

Simplified diagnostic procedure for obstructive sleep apnoea syndrome: lower subsequent compliance with CPAP. J. Krieger, E. Sforza, C. Petiau, T. Weiss. OERS Journals Ltd 1998. ABSTRACT: The aim of this study was to investigate whether a simplified diagnostic procedure based on ambulatory monitoring with MESAM IV altered subsequent compliance with continuous positive airway pressure (CPAP) in obstructive sleep apnoea (OSA) patients.

During a period of 16 months, 60 patients with symptoms evocative of OSA and positive MESAM recording were prescribed CPAP after titration with standard polysomnography. Compliance was followed during 2 yrs based on built-in time counters and was compared with the compliance in two comparison groups: an equal number of equally severely affected patients diagnosed with standard polysomnography during the 18 months ( 8 months before and 8 months after) preceding and following the study period and a group of 48 patients with an estimated similar apnoea/hypopnoea index but less typical clinical and/or MESAM features, diagnosed as having OSA based on polysomnography during the study period.

The three groups were not different by age, body mass index, or sleepiness score. Patients diagnosed with the ambulatory procedure had higher drop-out rates $(21.7 \%$ versus $10 \%$ and $6.25 \% ; \mathrm{p}<0.05)$ and lower rates of use of their CPAP $\left(4.3 \pm 0.3 \mathrm{~h} \cdot \mathrm{night}^{-1}\right)$ than any of the control groups $\left(5.3 \pm 0.2\right.$ and $\left.5.6 \pm 0.2 \mathrm{~h} \cdot \mathrm{night}^{-1}, \mathrm{p}<0.05\right)$.

In conclusion, there is a risk that ambulatory diagnostic procedures alter the relationship of patients to their disease and/or the medical staff so that subsequent compliance with treatment may be decreased. The greatest care concerning compliance should be taken before an ambulatory-based diagnostic procedure is implemented. Eur Respir J 1998; 12: 776-779.

Recent data have repeatedly shown that obstructive sleep apnoea (OSA) is a frequently encountered disorder, affecting about $5 \%$ of women and $10 \%$ of men in the middle-age range [1]. Therefore, it is not surprising that most sleep laboratories are overcrowded with patients presenting with features suggesting OSA (mainly somnolence, snoring and/or witnessed apnoeas).

Sleep laboratories are all faced with the problem of finding a compromise between full polysomnography in the first instance (which may result in unacceptably long waiting lists and is expensive) and cheap preselection examinations such as oximetry (which save time and money but which will miss an unacceptable proportion of sleep-disordered breathing and all other causes of somnolence [2]).

Recently, in an editorial in this journal, TESCHLER and BERTHON-JONES [3] proposed a compromise with the following steps: 1) clinical history and examination; 2) unattended home polysomnography including oximetry and semiquantitative flow if (1) is suggestive of OSA; 3) initiation of continuous positive airway pressure (CPAP) in a semiattended setting if (2) is positive; 4) if sleep-disordered breathing and desaturation are shown to be controlled with CPAP, home treatment is initiated; and 5) intensive outpatient follow-up.

A few years ago, the same considerations led to the use of an ambulatory-based diagnosis procedure in patients
Sleep Disorders Unit, Hôpitaux Universitaires de Strasbourg, France.

Correspondence: J. Krieger

Clinique Neurologique

F-67091 Strasbourg Cedex

France

Fax: 33388116343

Keywords: Ambulatory monitoring compliance

diagnostic procedure

obstructive sleep apnoea syndrome polysomnography

Received: February 91998

Accepted after revision July 81998 whose clinical features and ambulatory recording provided reasonable evidence for the diagnosis of obstructive sleep apnoea syndrome (OSAS). After 18 months, a decision was made to return to the previous sleep laboratory based procedure, not because of difficulties in the diagnosis but because of decreased compliance in the patients evaluated with the ambulatory-based procedure.

The present report evaluates the incidence of the ambulatory-based diagnostic strategy on subsequent compliance with CPAP by comparing compliance data in patients with ambulatory based diagnosis versus patients with standard laboratory polysomnography.

\section{Methods}

\section{Patients}

All patients underwent a clinical interview with and examination by an experienced sleep physician (JK, CP or ES) and were given a standardized questionnaire, followed by an ambulatory examination with MESAM IV during a single night. All MESAM IV recordings were scored by the same scorer $(\mathrm{JK})$.

Ambulatory-based diagnosis (direct continuous positive airway pressure). Between 1 October 1993 and 31 January 1995 , all patients with clear clinical symptoms of OSA 
(daytime sleepiness and heavy snoring and/or witnessed apnoeas) together with a positive ambulatory recording were included in the direct CPAP group. Because the difficulties of diagnosis based on MESAM recordings, which have been recently emphasized, with more than $40 \%$ of the MESAM diagnosis being equivocal [4, 5], no attempt was made to establish a sleep-disordered breathing index. Instead, only those patients who had an unequivocal recording, i.e. the typical pattern of OSA shown in figure 1 during at least $80 \%$ of the recording time, were included. These patients were admitted to hospital for an adaptation night followed by a CPAP titration night. The patients with a less typical MESAM pattern followed the usual procedure with standard polysomnography.

CPAP was prescribed based on ambulatory investigation in 60 patients (group 1: direct CPAP).

Comparison groups. As patient severity may play a critical role in compliance, the intention was to compare the direct CPAP group with groups of equivalent apnoea severity diagnosed with standard laboratory polysomnography. However, as the apnoea/hypopnoea index (AHI) was not available in the direct CPAP group, it was hypothesized that the recruitment was stable in patient severity over time and, as the selection criteria for the direct CPAP group obviously selected the most severe cases among the patients who were prescribed CPAP over this period, a patient group (group 2) who had been prescribed CPAP during an equivalent period was selected by adjusting the inclusion AHI threshold so that it provided a sample of equivalent size. The AHI which fulfilled this criterion was 72.5 and the selected comparison group had an AHI of $97.6 \pm 17.4($ mean \pm SD). The comparison period was of an equivalent duration, including 8 months before 1 October 1993 and 8 months after 31 January 1995.

A second comparison group (group 3) comprised patients who were prescribed CPAP between 1 October 1993 and 31 January 1995 whose AHI was comprised within $97.6 \pm 2 \mathrm{sD}$, i.e. \pm 34.8 , but who had followed the usual procedure with standard polysomnography. These patients had not been included in the direct CPAP protocol, because distance $(>100 \mathrm{~km})$ precluded ambulatory investigations, because of less typical case histories or because of less conclusive MESAM data (technical failures or underestimation of sleep-disordered breathing).

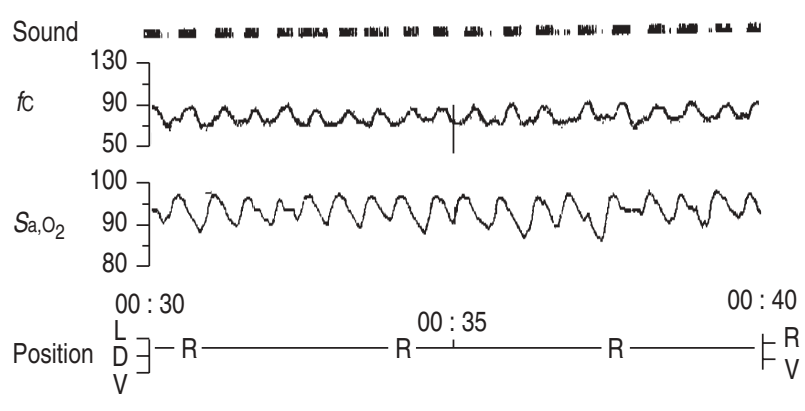

Fig. 1. - Typical example of positive MESAM IV recording: repeated interruption of snoring on the sound channel, in association with oscillations in cardiac frequency $(f \mathrm{C})$ and arterial oxygen saturation $\left(\mathrm{Sa}_{\mathrm{a}} \mathrm{O}_{2}\right)$; the patient was in the right lateral position $(\mathrm{R})$ during this sequence. Observation time shown (min). L: left lateral position; D: dorsal (supine) position; V: ventral (prone) position.
The two comparison groups followed exactly the same diagnostic and treatment procedures as the direct CPAP group, except that they spent one more day in hospital, with a standard polysomnography on the second night, before the CPAP titration night.

All patients. Before the titration night, all patients were interviewed by a senior member of the medical staff (ES or JK). The results of the diagnostic procedure were shown to the patient and all explanations regarding the treatment were given.

In all patients, the same protocol was followed during the titration night: CPAP was progressively increased starting with the smallest possible pressure of $3 \mathrm{cmH}_{2} \mathrm{O}$. The steps were $1 \mathrm{cmH}_{2} \mathrm{O}$ every minute until apnoeas and hypopnoeas were eliminated, and $1 \mathrm{cmH}_{2} \mathrm{O}$ every 5 min until snoring was eliminated and oesophageal pressure swings did not exceed twice their value during quiet wakefulness. In many instances, snoring and hypopnoeas (or at times obstructive apnoeas) reappeared during the course of the night, which required an additional increase in CPAP.

After the titration night, an additional day and night in the hospital was devoted to respiratory function tests and blood gas analysis, as well as adaptation to the CPAP mask and patient education.

The patients were then sent home with their machine and followed by their home-care provider (all affiliated to the French homecare network ANTADIR: ADIRAL for 150 patients, ARAIRLOR for 13 and AIR for five) including technicians, nurses and physicians who provided outpatient support. Patients were followed up at the outpatient Sleep Clinic 3 months later and 1 yr later, except if a specific problem required more frequent intervention.

All patients had been informed after the initial interview that some of the data in their medical charts might be used for research purposes, and all gave informed written consent. As the investigation required no specific intervention different from usual clinical procedures, no approval of an Ethics Committee was considered necessary.

\section{Methods}

Sleepiness scores. In each questionnaire, the patients were asked to report how frequently (never $=1$, sometimes $=2$, often $=3$, almost always $=4$, does not know or not concerned by the given activity $=0$ ) they dozed off during seven activities of daily life (after a meal, at work, at social meetings, watching TV, while reading, when a passenger in a car or a means of transportation, or while driving). A sleepiness score was computed as the average score of the answers, provided the patients had given an answer different from 0 to at least four items; otherwise, the sleepiness score was considered missing. This sleepiness score is close in its principle to the Epworth Sleepiness score and it was shown to separate OSA from normal subjects [6] and to decrease with CPAP treatment [7]. Sleepiness scores could be calculated in 166 patients, with a missing score in one patient in group 1 and one patient in group 3 .

Compliance. Compliance with CPAP was evaluated based on the readings of the time counters built in the CPAP 
devices, by dividing the difference between two consecutive readings by the number of days elapsed. All compliance data were collected 2 yrs after the initiation of CPAP treatment, so that all patients had the same follow-up duration.

\section{Statistical analysis}

Comparisons between groups were made using one-way analysis of variance (ANOVA) followed by the post hoc Student-Newman-Keuls test. Proportions were compared using the Chi-squared test. Differences were considered significant for $\mathrm{p}<0.05$. Results are reported as means \pm SEM except when stated otherwise.

\section{Results}

There was no statistically significant difference in any of the variables analysed between the two subgroups of patients treated with CPAP during the 8 months before 1 October $1993(n=18)$ and after 31 January $1995(n=42)$; therefore, these patients were pooled into a single group (group 2, $\mathrm{n}=60$ ). The main characteristics of the 60 patients (group 1) who followed the direct CPAP procedure during the study period (16 months) are given in table 1 , together with those of the 60 most severe patients treated with CPAP before and after the study period (group 2, n= 60 ) and the 48 patients whose AHI fell within $97.6 \pm 35$ who were prescribed CPAP during the study period (group 3 ) following the usual procedure including standard polysomnography.

Before treatment, there were no differences between the direct CPAP group and any of the comparison groups in any of the investigated variables (table 1). The two comparison groups differed from each other in that the less typical patients who were prescribed CPAP during the study period (group 3) were slightly less overweight and had higher daytime arterial oxygen tension than the comparison group who started CPAP before and after the study period (group 2).

Table 1. - Main characteristics of the three groups of patients

\begin{tabular}{|c|c|c|c|c|}
\hline & Group 1 & Group 2 & Group 3 & Sig. \\
\hline Age yr & $56.7 \pm 1.4$ & $54.0 \pm 1.4$ & $55.6 \pm 1.4$ & NS \\
\hline AHI events.h-1 & - & $97.7 \pm 2.5$ & $101.8 \pm 3.0$ & NS \\
\hline BMI $\mathrm{kg} \cdot \mathrm{m}^{-2}$ & $33.1 \pm 0.8$ & $34.4 \pm 0.8$ & $31.4 \pm 0.9$ & $2 v s 3$ \\
\hline$P \mathrm{a}, \mathrm{O}_{2} \mathrm{mmHg}$ & $74.5 \pm 1.3$ & $70.0 \pm 1.2$ & \pm 1.0 & $2 v s 3$ \\
\hline $\mathrm{Pa}_{\mathrm{a}, \mathrm{CO}_{2}} \mathrm{mmHg}$ & $43.3 \pm 0.6$ & $42.9 \pm 0.6$ & $42.5 \pm 0.6$ & NS \\
\hline Sleepiness score & $8.8 \pm 0.4$ & 9.2 & 0.4 & NS \\
\hline AHI/CPAP & $6.0 \pm 0.8$ & $6.1 \pm 0.8$ & $6.8 \pm 0.8$ & NS \\
\hline CPAP & $9.8 \pm 0.3$ & $9.8 \pm 0.3$ & $9.7 \pm 0.4$ & NS \\
\hline Rates of use $h \cdot n i g h t-1$ & $4.3 \pm 0.3$ & $5.3 \pm 0.2$ & $5.6 \pm 0.2$ & $1 v s 2,3$ \\
\hline
\end{tabular}

Group 1: patients diagnosed based on ambulatory recording (MESAM IV); group 2: patients diagnosed based on standard polysomnography before and after the study period; group 3 : patients diagnosed based on standard polysomnography during the study period. AHI: apnoea/hypopnoea index; BMI: body mass index; $\mathrm{Pa}, \mathrm{O}_{2}$ : arterial oxygen tension; $\mathrm{Pa}, \mathrm{CO}_{2}$ : arterial carbon dioxide tension; CPAP: continuous positive airway pressure; AHI/CPAP: AHI during the CPAP titration night. Sig.: Statistical significance $(\mathrm{p}<0.05)(1 \mathrm{mmHg}=0.133 \mathrm{kPa}$. $)$
These were obviously patients with severe OSA, by definition, as the direct CPAP treatment was applied only to those patients whose clinical features as well as the results of MESAM IV recordings were unambiguous.

The number of apnoeas and hypopnoeas recorded during the titration night (corresponding to the period before efficient CPAP was reached and to episodes of reappearance of events during the course of the night) did not differ between the three groups, nor did the level of CPAP applied during home treatment (table 1).

Of the 60 patients who started CPAP following the direct procedure, 41 were still on CPAP 2 yrs later, 13 quit CPAP, three died and three continued their treatment in another centre. Of the 60 patients who started CPAP just before or just after the experimental direct CPAP period, 48 were still on CPAP 2 yrs later, six quit CPAP, two died, two were considered cured (on a clinical and polysomnographic basis) after weight loss and two continued their treatment in another centre. Of the 48 patients who received CPAP during the experimental period but followed the usual procedure with standard polysomnography, 41 were still on CPAP 2 yrs later, three quit CPAP, three died and one continued his treatment in another centre. The percentage of patients who quit CPAP was significantly different between groups $(21.7 \%$ in group 1 versus $10 \%$ in group 2 and $6.25 \%$ in group $3 ; \mathrm{p}<0.05)$.

Measures of the rate of use of CPAP (from the time counters) were available in 54 patients in group 1, 58 in groups 2 and all patients in group 3 , as six patients in group 1 and two patients in group 2 had quit CPAP before the first reading (within 3 months of initiation of treatment) had been taken. Table 1 shows the average rate of use after 2 yrs (or at the time of treatment interruption). There was no difference in the mean rate of use between the two comparison groups, whereas the direct CPAP group had significantly lower rates of use than groups 2 and 3 .

\section{Discussion}

These data show that patients who were prescribed CPAP for OSA diagnosed on an ambulatory recording basis, without a standard polysomnography, had lower compliance rates with CPAP than patients prescribed CPAP after standard polysomnography, in spite of presumably equivalent severity (in terms of the AHI index) and similar age, BMI, daytime blood gases and sleepiness score.

Another study has suggested that the diagnostic procedure may influence subsequent compliance with CPAP. In a prospective multicentre 1-yr follow-up study which investigated 547 patients, it was shown that patients diagnosed with ventilatory polygraphy had higher drop-out rates than patients diagnosed with full polysomnography (available on request from ANTADIR, 66 Boulevard SaintMichel, 75006 Paris, France).

Several factors may explain these findings. As the patients in the direct CPAP group spent less time in hospital, less time was available for patient education, which has been shown to play a critical role in subsequent compliance with CPAP [8]. Beyond education per se, a positive relationship between the patient and the medical staff may play a critical role. Spending less time in hospital shortens the time available for the establishment of such a relationship. 
Limited diagnostic procedures provide less explicit pictures of the breathing abnormalities occurring during sleep; therefore, it is more difficult to show the patients the importance of their disorder. This is especially relevant for sleep apnoea patients, who often deny their symptoms and are reluctant to accept that they are ill and that their condition requires treatment, as most of the abnormalities occur during sleep.

It is also possible that the medical staff were less confident in a diagnosis based on an ambulatory procedure that differed from the usual in-hospital polysomnographybased procedure and were, thus, less convincing with the patients.

Whatever the factors causing the decreased compliance, which can only be speculated upon from the results of the present study, the data suggest that a simplified diagnostic procedure may cause lower compliance with treatment. The greatest care should be taken when implementing ambulatory procedures, as the cost of reduced compliance may be more than the savings on the diagnostic procedure.

\section{References}

1. Young T, Palta M, Dempsey J, Skatrud J, Weber S, Badr $\mathrm{S}$. The occurrence of sleep-disordered breathing among middle-aged adults. $N$ Engl J Med 1993; 328: 1230 1235.

2. Ferber R, Millman R, Coppola M, et al. Portable recording in the assessment of obstructive sleep apnea. Sleep 1994, 17: 378-392.

3. Teschler H, Berthon-Jones M. Full polysomnography versus home sleep study: searching for the optimal procedure. Eur Respir J 1997; 10: 1699-1700.

4. Parra O, Garcia-Esclasans N, Montserrat JM, et al. Should patients with sleep apnoea/hypopnoea syndrome be diagnosed and managed on the basis of home sleep studies? Eur Respir J 1997; 10: 1720-1724.

5. Whittle AT, Finch SP, Mortimore IL, MacKay TW, Douglas NJ. Use of home sleep studies for diagnosis of the sleep apnoea/hypopnoea syndrome. Thorax 1997; 52: 1068-1073.

6. Petiau C, Delanoë C, Hecht MT, Chamouard V, Krieger J. Somnolence et syndrome d'apnées du sommeil. Analyse de 188 questionnaires (102 malades et 86 témoins). Neurophysiol Clin 1993; 23: 77-85.

7. Sforza E, Krieger J. Daytime sleepiness after long-term nasal continuous positive airway pressure (CPAP) treatment in obstructive sleep apnea syndrome. J Neurol Sci 1992; 110: 21-26.

8. Chervin RD, Theut S, Bassetti C, Aldrich MS. Compliance with nasal CPAP can be improved by simple interventions. Sleep 1997; 20: 284-289. 